\title{
Carnets
}

Revue électronique d'études françaises de l'APEF

Deuxième série - 15 | 2019

Jules Verne et les pouvoirs de l'imagination

\section{Frritt-Flacc au Brésil : la narration visuelle}

\section{María-Lourdes Cadena et María-Teresa Barea}

\section{(2) OpenEdition}

Journals

Édition électronique

URL : http://journals.openedition.org/carnets/9437

DOI : 10.4000/carnets.9437

ISSN : 1646-7698

Éditeur

APEF

\section{Référence électronique}

María-Lourdes Cadena et María-Teresa Barea, « Frritt-Flacc au Brésil : la narration visuelle », Carnets [En ligne], Deuxième série - 15 | 2019, mis en ligne le 31 janvier 2019, consulté le 30 avril 2019. URL: http://journals.openedition.org/carnets/9437 ; DOI : 10.4000/carnets.9437

Ce document a été généré automatiquement le 30 avril 2019.

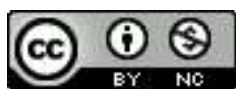

Carnets est mis à disposition selon les termes de la licence Creative Commons - Atribution - Pas d'utilisation commerciale 4.0 International. 


\title{
Frritt-Flacc au Brésil : la narration visuelle
}

\author{
María-Lourdes Cadena et María-Teresa Barea
}

\section{NOTE DE L'AUTEUR}

T3AxEL, groupe de recherches de l'Université de Saragosse (Espagne). Financement :

Gouvernement d'Aragon-FEDER.

1 Cette nouvelle de Jules Verne, parue pour la première fois dans Le Figaro illustré en 1884, et après en livre en 1886, dans la maison d'édition Hetzel, n'a pas vu le jour au Brésil jusqu'au xxI ${ }^{e}$ siècle. Selon les responsables de la maison d'édition Pulo do Gato, le récit était resté inédit dans ce pays jusqu'en 2012 quand ils se sont décidés à publier cette première traduction en portugais « après avoir fait le tour du monde en 80 jours en ballon jusqu'à découvrir le conte Frritt-Flacc.»(Verne, $2012: 72)^{1}$.

Parler d'images, c'est mettre en relief ce que la représentation visuelle ou mentale de quelque chose transmet au récepteur. Les illustrations sont des représentations visuelles de nature graphique ou picturale dont la fonction essentielle sert à amplifier, compléter, décrire ou prolonger un texte donné. D'un point de vue sémiologique, les illustrations seraient donc des images associées à des mots étant toujours subordonnées au texte ${ }^{2}$.

Dans la narration le narrateur, créé par l'auteur, raconte des faits réels ou imaginaires. La narration littéraire est organisée dans un cadre narratif, avec une situation principale et d'autres secondaires dont les actions se combinent entre elles, et dont les développements s'entremêlent. Cela conforme le rythme de la narration et permet d'aboutir à la situation finale. En outre, les personnages, fil conducteur des faits, sont un autre élément fondamental. C'est à leurs gestes, voix, pensées et actions de faire avancer ou ralentir le récit. De même, le cadre physique où les personnages évoluent, et l'atmosphère où les actions se déroulent, sont très importants.

D'après Mihaela Radulescu (2014), dans la narration visuelle, le texte et l'image sont mis en interaction dialogique et l'observateur est amené à comparer non seulement les 
formes et les contenus du texte et de l'image, mais doit aussi tenir compte de la signification née de la relation générée entre ces deux composantes. D'ailleurs, l'illustrateur, l'artiste, est chargé de rapporter sous forme visible, de réinterpréter en images ce que la narration littéraire propose. Dans les albums illustrés, les images et les mots sont, en principe, fusionnés. Le narrateur serait à la fiction littéraire ce que l'illustrateur serait à la perspective visuelle.

Dans ce travail, nous nous pencherons sur deux parutions brésiliennes assez originales de cette nouvelle de Verne, la plus récente en 2016, et l'autre en 2012. On examinera surtout leur partie visuelle et picturale, cet autre langage qui pourrait compléter l'œuvre originale française et nous permettre ainsi de mieux la connaitre.

Il ne s'agit pas de deviner ce que les concepteurs ou les auteurs de ces adaptations ont voulu transmettre avec leurs représentations visuelles. C'est plutôt l'interprétation personnelle que l'observateur fait à travers la narration visuelle que ces créateurs nous proposent.

La singularité de ces deux publications réside dans le format et dans leur manière d'expression: d'une part une application mobile animée et d'autre un livre fabulé et illustré.

Frritt-Flacc est un récit bref qui n'a que sept chapitres et environ deux mille quatre cents mots, conçu pour un public adulte, mais qui pouvait être lu par tout type de lecteurs.

Dans ces deux versions brésiliennes, le public-cible est majoritairement jeune, formé par des enfants et des adolescents; un groupe de population qui aime le visuel et se débrouille bien avec la technologie.

L'histoire de Frrit-Flacc est bien connue: dans la ville imaginaire de Luktrop habite le docteur Trifulgas, un homme caractérisé par sa cupidité et son avarice. De l'autre côté de la ville, à Val Karniou, un homme est en train de mourir : la fille, la femme et la mère du moribond, à tour de rôle, frappent à la porte du docteur pour lui demander de les aider. Finalement, en échange d'une bonne quantité d'argent, il décide d'y aller. Sous la tempête, contre le vent (Frritt) et sous la pluie (Flacc) le trajet entre Luktrop et Val Karniou, est truffé, jalonné de bruits, de sonorités, d'allitérations. La nouvelle traite le thème du double et le récit est organisé à partir de la phrase finale : ce médecin qui finalement a accepté d'aller soigner le moribond «se meurt entre ses propres mains. » L'illustrateur et le créateur d'images animées doivent répondre au défi d'adapter de la meilleure manière le récit littéraire qu'ils ont devant eux. Conformément aux particularités de Frritt-Flacc ébauchées par Carmelina Imbroscio (2013), que nous assumons pleinement, l'établissement de l'intrigue en images devra tenir compte de quelques caractéristiques littéraires comme l'utilisation d'un ton ironique, un langage dégagé et un rythme soutenu ; les assonances (du latin adsonare, c'est-à-dire, répondre à un son par un autre son), les allitérations (qui provoquent des effets sonores et expressifs), et les onomatopées suggestives, pour arriver à obtenir une harmonie imitative; une contrée imaginaire et sinistre, les éléments naturels qui se déchainent dans une nuit lugubre contre le vent et sous la pluie, un volcan dominant le village et vomissant des flammes rougeâtres, des cloches qui sonnent mises en branle par l'ouragan; un homme avare, une mort annoncée dans un contexte "gothique», une atmosphère sinistre qui suscite de fortes émotions, comme la peur de Trifulgas,

(...) les repères spatiaux et temporels s'embrouillent (il a avancé longuement et durement dans la tourmente pour se retrouver au point de départ) (...) ses sens sont en proie à une hallucination autoscopique (il aperçoit son sosie moribond dans son lit), (...). (Imbroscio, $2013: 91$ ). 


\section{Frritt-Flacc animé}

Nous allons commencer par l'analyse de l'application mobile pour smartphone et tablette, téléchargeable gratuitement, dont le texte, curieusement ne peut pas être lu en français, uniquement en portugais, espagnol et anglais ${ }^{3}$.

Selon la Chaire de lecture de l'Unesco, c'est le seul livre numérique placé entre les 10 meilleurs livres de $2016^{4}$. C'est aussi le premier livre-app d'animation de la collection Nova Perspectiva, un projet réalisé en collaboration entre Novozymes, StoryMax et SESIPR (Service social de l'industrie de Parana au Brésil), supporté par CRBio (Conseil régional biologique de Parana). Il s'agit donc d'un projet avec des objectifs littéraires, éducatifs et sociaux.

À travers les paroles -en anglais- de son vice-président pour l'Amérique latine, la société Novozymes explique sa curieuse vision de la trame de Frritt-Flacc: son intrigue, sous forme de suspense, tourne autour des différences sociales, abordant l'indifférence humaine au problème ainsi que la punition de la pauvreté de nos jours ${ }^{5}$.

Donc, la nouvelle de Verne a été choisie par ses concepteurs pour attirer l'attention sur l'ODD1 (premier objectif de développement durable des Nations Unies) qui propose l'éradication de la pauvreté et insiste sur le fait que la Biologie peut transformer notre planète en un monde meilleur pour tous.

D'autre part, StoryMax est une société, une startup dans le domaine de la littérature digitale, avec une trajectoire de plus de dix ans dans le marché de l'édition et des études dans l'Éducommunication. StoryMax a été reconnue non seulement au Brésil, mais dans le monde entier avec des prix éducatifs, technologiques, de lecture, et d'innovation ${ }^{6}$. C'est aussi une application qui réunit des histoires publiées pour enfants et adolescents dans un format audiovisuel et interactif, parmi lesquelles deux sont de Jules Verne, Nautilus (basée sur Vingt mille lieues sous les mers ${ }^{7}$ ) et Frritt-Flacc.

La bande annonce de ce livre-app, qui ne dure qu'une minute et demie, est très bien conçue pour que le lecteur puisse se faire une idée de l'adaptation animée ${ }^{8}$, les couleurs, les effets sonores et l'interactivité.

L'animation choisie par les graphistes est en 2D, avec des mouvements simplifiés, où les éléments se déplacent horizontalement (de gauche à droite) et verticalement (de haut en bas). Les objets sont plats, sans texture, clairs, aux contours bien délimités; et pour donner une certaine idée de profondeur parfois le paysage est vu en plongée. Le type de graphisme convient très bien à cette histoire fantastique parce que la ressemblance de l'animation 2D avec la réalité est très faible.

La navigation utilise un crâne, une tête de mort pour se déplacer, et outre le texte, elle inclut des activités et des contenus additionnels ${ }^{9}$. On propose des activités de production écrite, des expériences scientifiques et d'action sociale, dans le but de sensibiliser les jeunes à l'éradication de la pauvreté et au développement durable. Parfois le rapport entre Frritt-Flacc et les activités éducatives proposées se montre peu naturel, voire forcé. Par exemple, sous la rubrique Activités, après la description du processus de la mort de Trifulgas, point culminant de la nouvelle où le médecin meurt comme beaucoup de pauvres gens qu'il a refusé d'aider, l'activité proposée nous invite à réfléchir sur la manière dont les décisions que l'on peut prendre ont de l'influence dans la vie des personnes sans moyens économiques. 
Esse trecho é um dos pontos altos do conto Frritt-Flacc, quando o ganancioso Trifulgas recebe o mesmo fim de muitos pobres a quem negou atendimento. Reflita sobre como as decisões que tomamos podem impactar a vida de pessoas menos favorecidas próximas de nós.

7 Certes, c'est pleinement dans la ligne de l'ODD1 et de l'interprétation de l'intrigue que font les concepteurs de l'app en montrant des personnages habituels dans n'importe quelle société : une famille pauvre et un médecin qui a oublié le rôle humaniste de sa profession.

La musique que l'on écoute tout au long de la lecture est la Lacrimosa, partie finale de la Sequentia Dies irae, Requiem en D minor de Mozart.

Comme nous avons déjà mentionné, l'utilisateur peut lire le texte en trois langues, mais aussi changer la force du vent, de la pluie, faire sonner la cloche du clocher, activer le volcan, ouvrir et fermer des fenêtres, pousser les nuages et même, essayer de ressusciter le mort.

Les couleurs utilisées dans le graphisme sont à remarquer, bien que le chromatisme et son symbolisme s'insère dans l'imaginaire collectif du monde occidental. À part le blanc, utile pour attirer l'attention sur des faits ou des personnes, et le noir, qui sert à marquer le mystère, le silence, la nuit, le mal, la mort et le deuil, le reste des couleurs sont dans la gamme du rouge, couleur agressive, efficace à montrer le sang et le feu, le danger, la violence et la colère. Le rouge vif convient à la maison du Six-Quatre et sert à attirer, sur elle, l'attention de l'observateur. En contrebalance on a du marron qui suggère l'âge, les objets anciens, le bois et le réalisme et, en complément, l'ocre ${ }^{10}$.

On peut dire que cette application a été créée avec un design monochromatique, et les tonalités qui prédominent vont du rouge violet (rouge bordeaux) au rose saumon et un rouge rouille, grenat, qui combine avec le marron.

Trifulgas (fig. 1) ${ }^{11}$ est présenté comme une personne âgée, chauve, habillée en noir, le regard pervers et une attitude posturale qui indique son avarice, sa cupidité. Uniquement la barbe et la moustache sont légèrement colorées, dans la gamme des rouges dégradés avec une pincée de violet, couleur de la fantaisie, des états de somnolence, de cauchemar, de folie.

Fig. 1

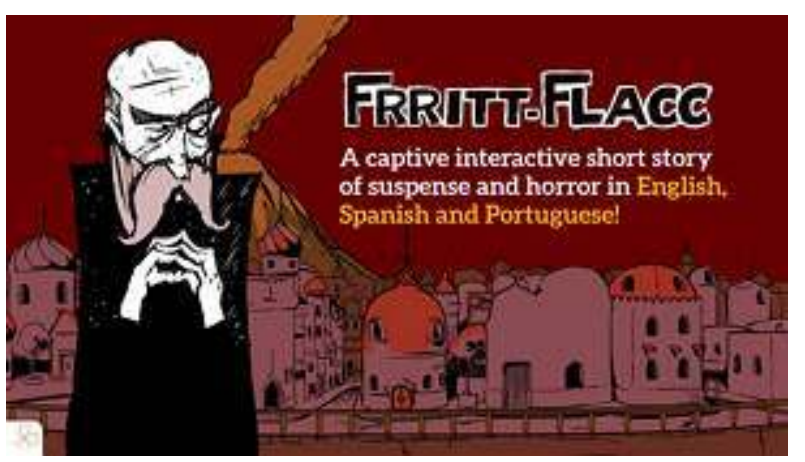

9 À titre d'exemple, nous avons choisi les deux instants les plus intéressants de ce livre, qui nous permettront de comparer la narration textuelle originale et la narration visuelle de l'application : la rencontre avec le double et le moment de la mort.

Ce premier moment de la rencontre fut illustré par George Roux dans l'édition de 1886 chez Hetzel (figure 2). 
Fig. 2

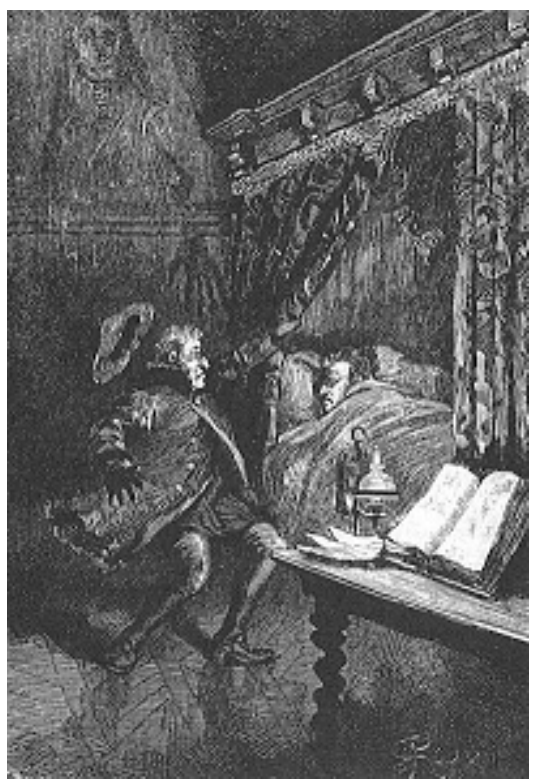

La situation est la suivante : Trifulgas est chez le moribond, Vort Kartif, et dans la lumière vague, il reconnait sa propre chambre, le mur en pierre, le même lit à baldaquin, les rideaux pareils, le mobilier, le Codex ouvert ; tout ceci est identique au sien. Et il est sur le point d'ouvrir les rideaux du lit du malade.

Les images des figures 3 à 6 de l'application illustrent le même instant.

Fig. 3

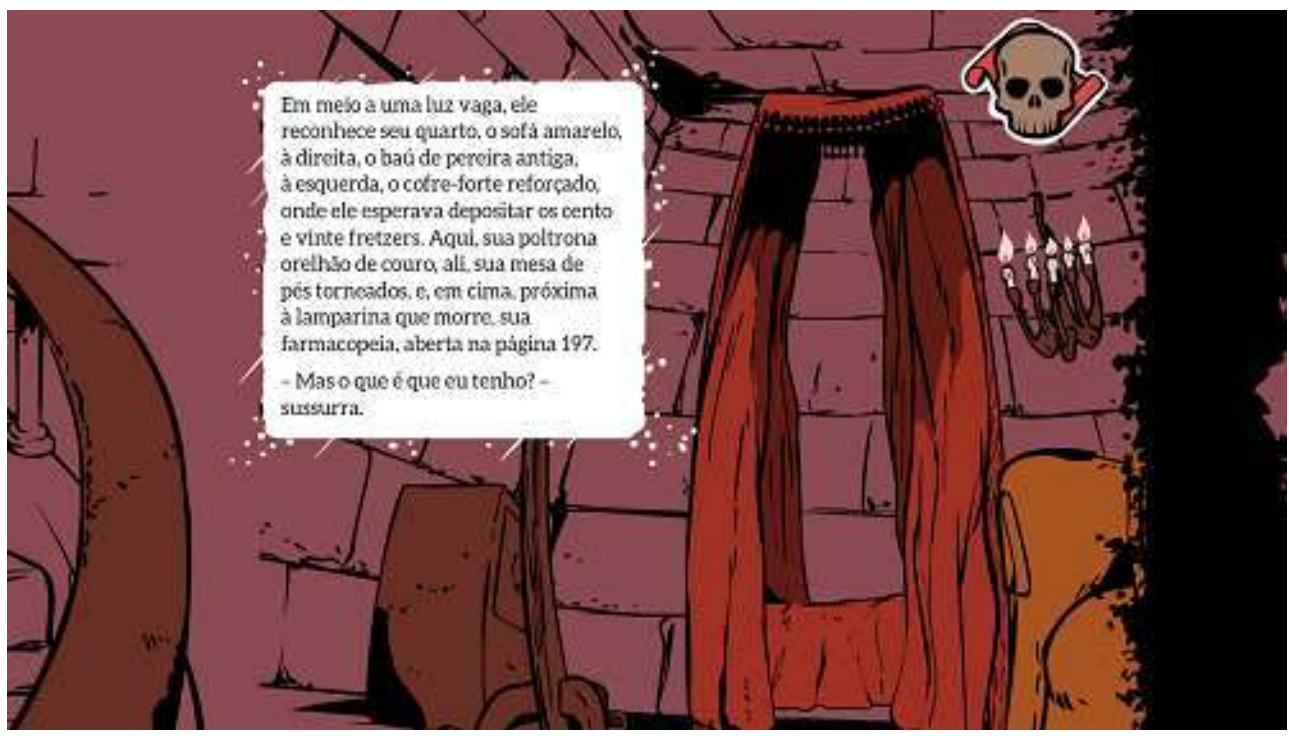


Fig. 4

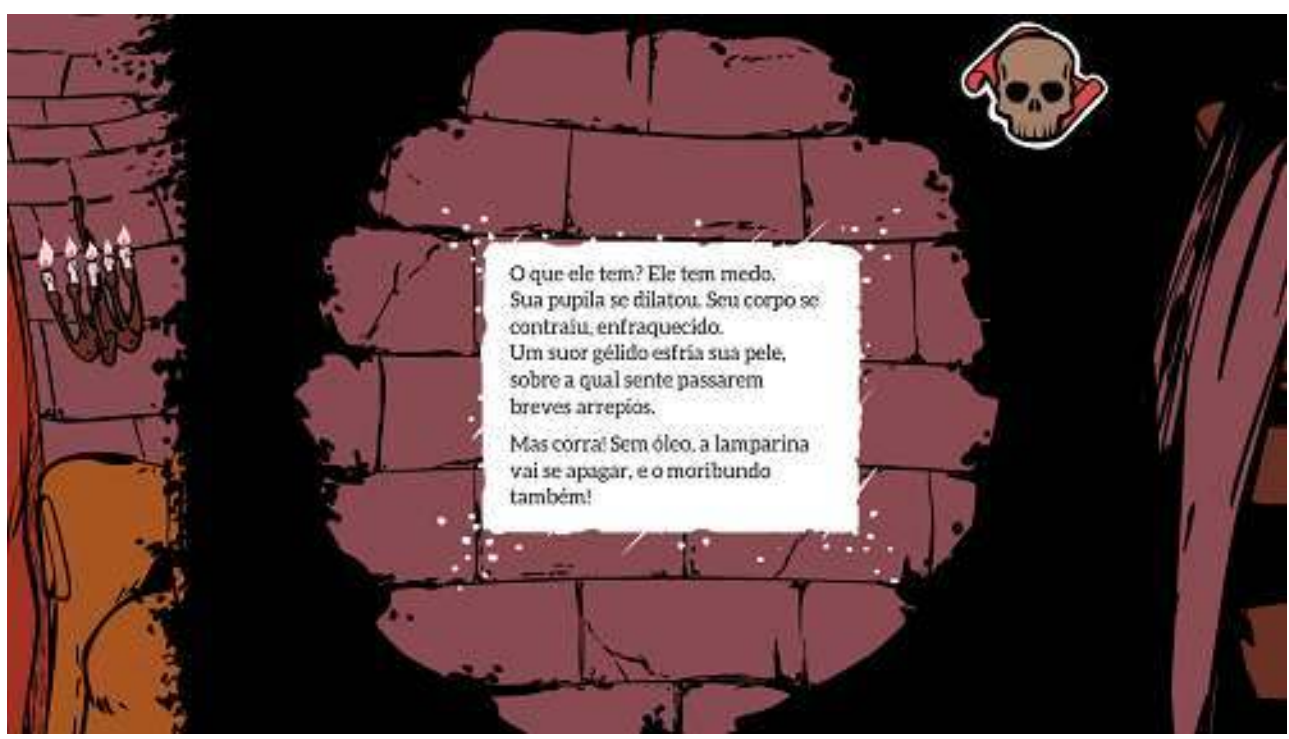

On entend un son percutant, comme les battements du cœur. Un mur en pierre est montré à travers un cercle noir (figure 4). Cela représente la position de l'observateur : il est au lit et à travers un orifice ouvert entre les rideaux du lit, il observe le mur en face. Nous, nous sommes le moribond. Les concepteurs de l'app nous ont mis à la place de Vort Kartif pour accentuer l'angoisse du moment.

Voilà, le spectateur est au lit. Et de l'autre côté des rideaux, le docteur, en premier plan est sur le point de les ouvrir (figure 5): «D'une main qui tremble, le docteur Trifulgas saisit les rideaux. Il les ouvre, il regarde » (chapitre vI).

Fig. 5

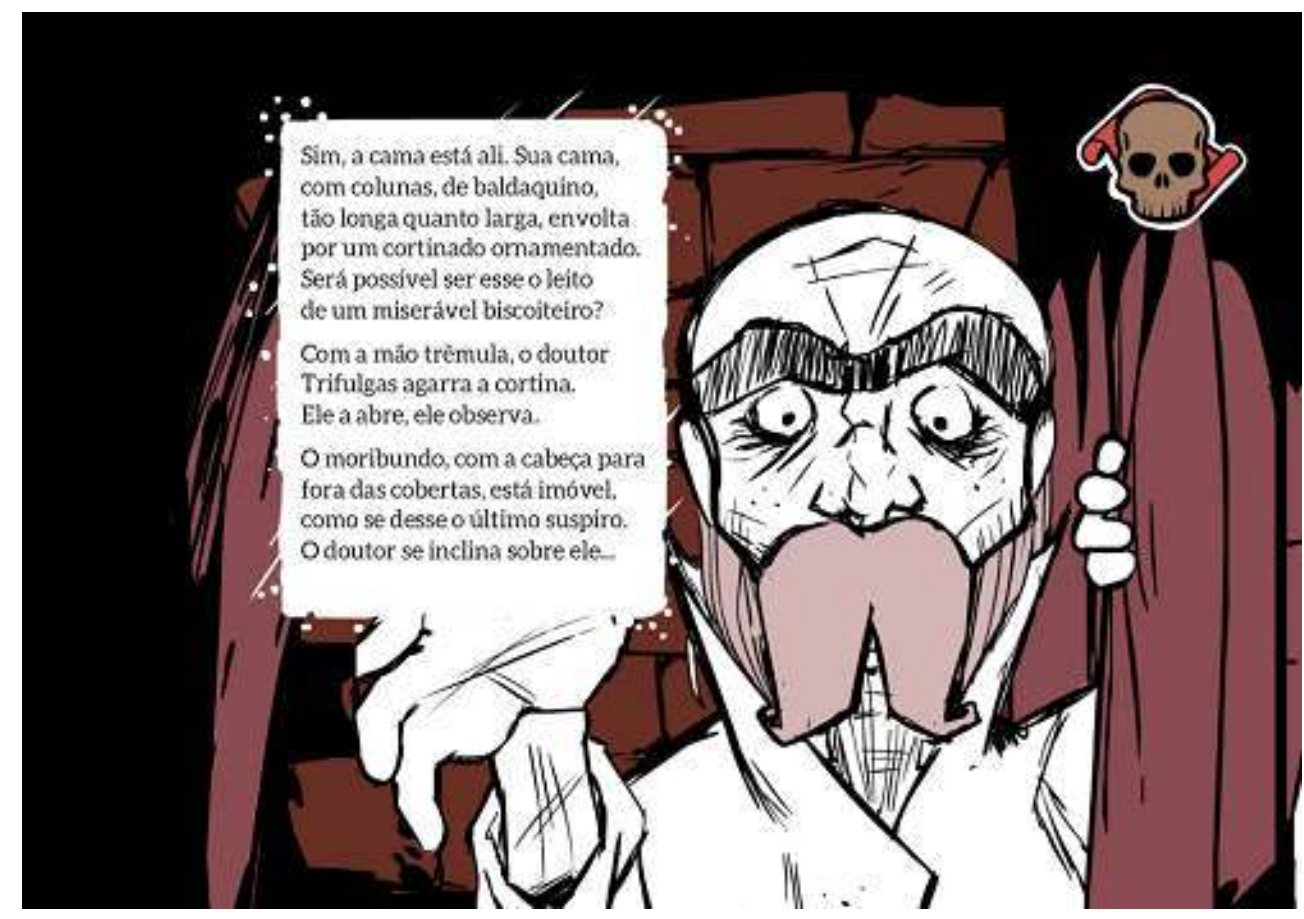


12 Au click sur l'écran, on entend une musique de film d'horreur. Le docteur ouvre un peu plus et laisse voir son étonnement et sa terreur (figure 6).

Fig. 6

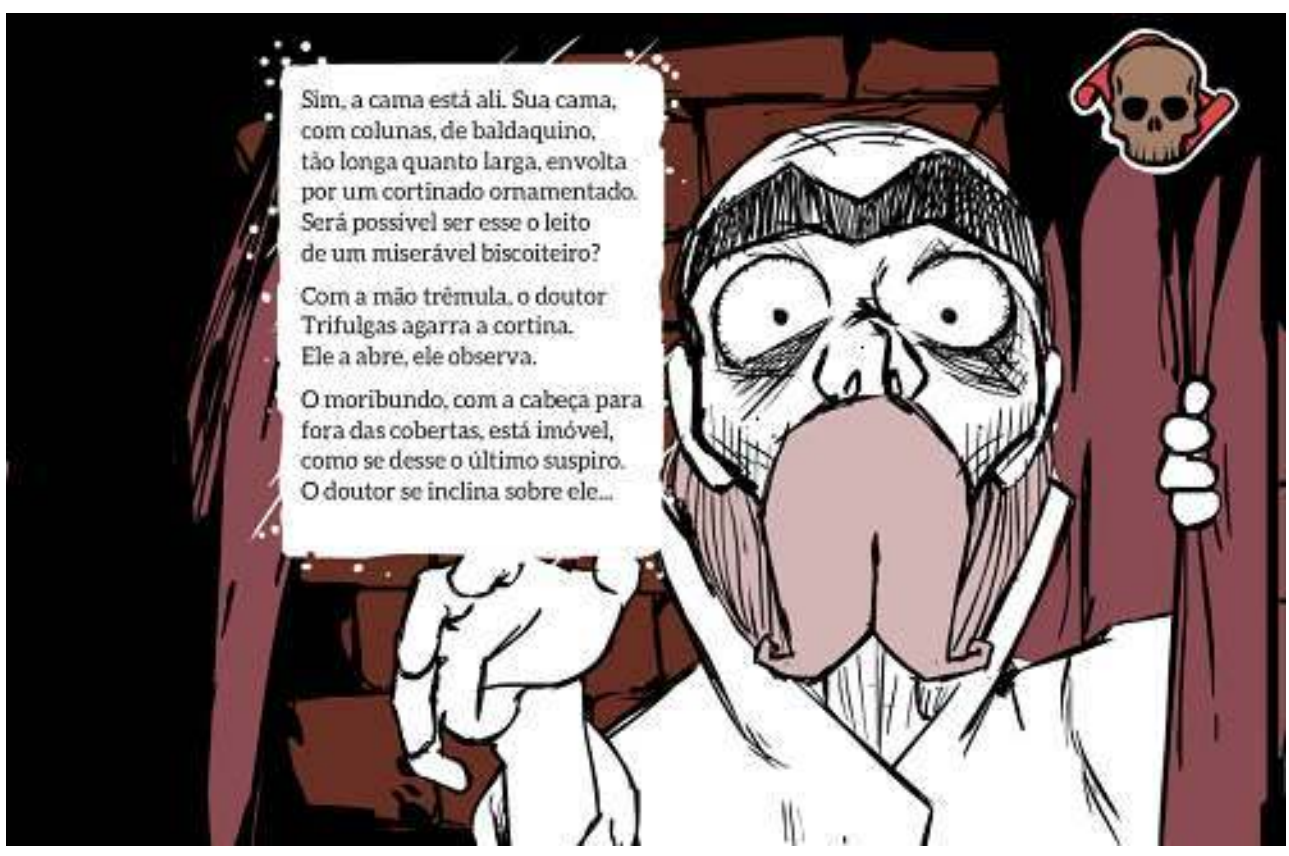

13 Bien que le moment de la mort de Trifulgas-Vort Kartif n'ait pas été illustré dans les éditions du XIX ${ }^{e}$ siècle, dans le Frritt-Flacc animé l'observateur est cependant placé debout sur la scène, sous le même angle que le cupide médecin. Tout est en silence. Le spectateur ne voit que les mains du docteur qui bougent. Derrière Trifulgas, le spectateur regarde en plongée le moribond couché sur son lit (figure 7). 
Fig. 7

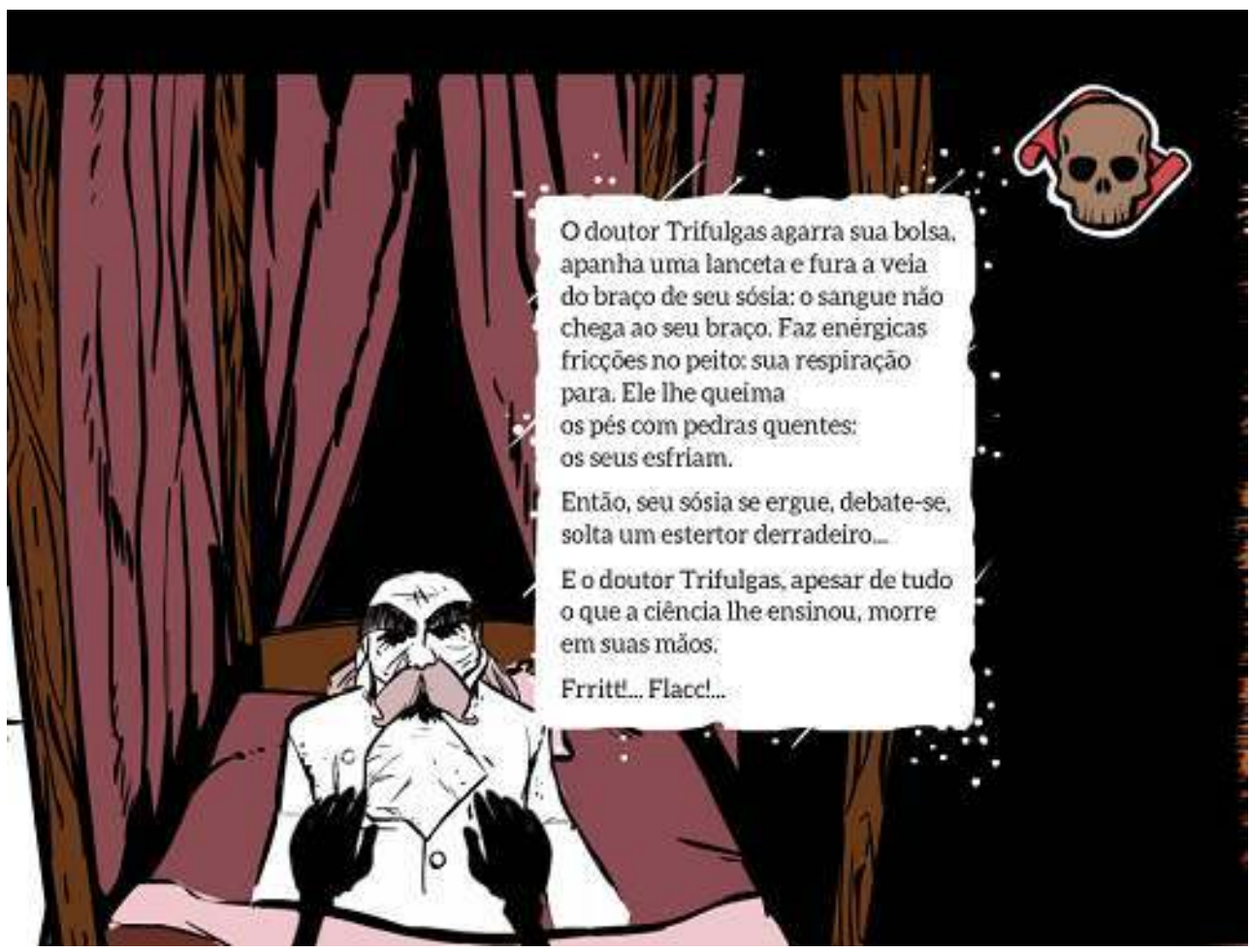

En cliquant sur l'écran, le moribond, les yeux grand ouverts, lance son dernier soupir, mais il est trop tard! On n'a pas pu le ressusciter (figure 8). «Et le docteur Trifulgas, malgré tout ce qu'a pu lui inspirer la science, se meurt entre ses mains. Frritt ! ... Flacc!» (chapitre VI).

Fig. 8

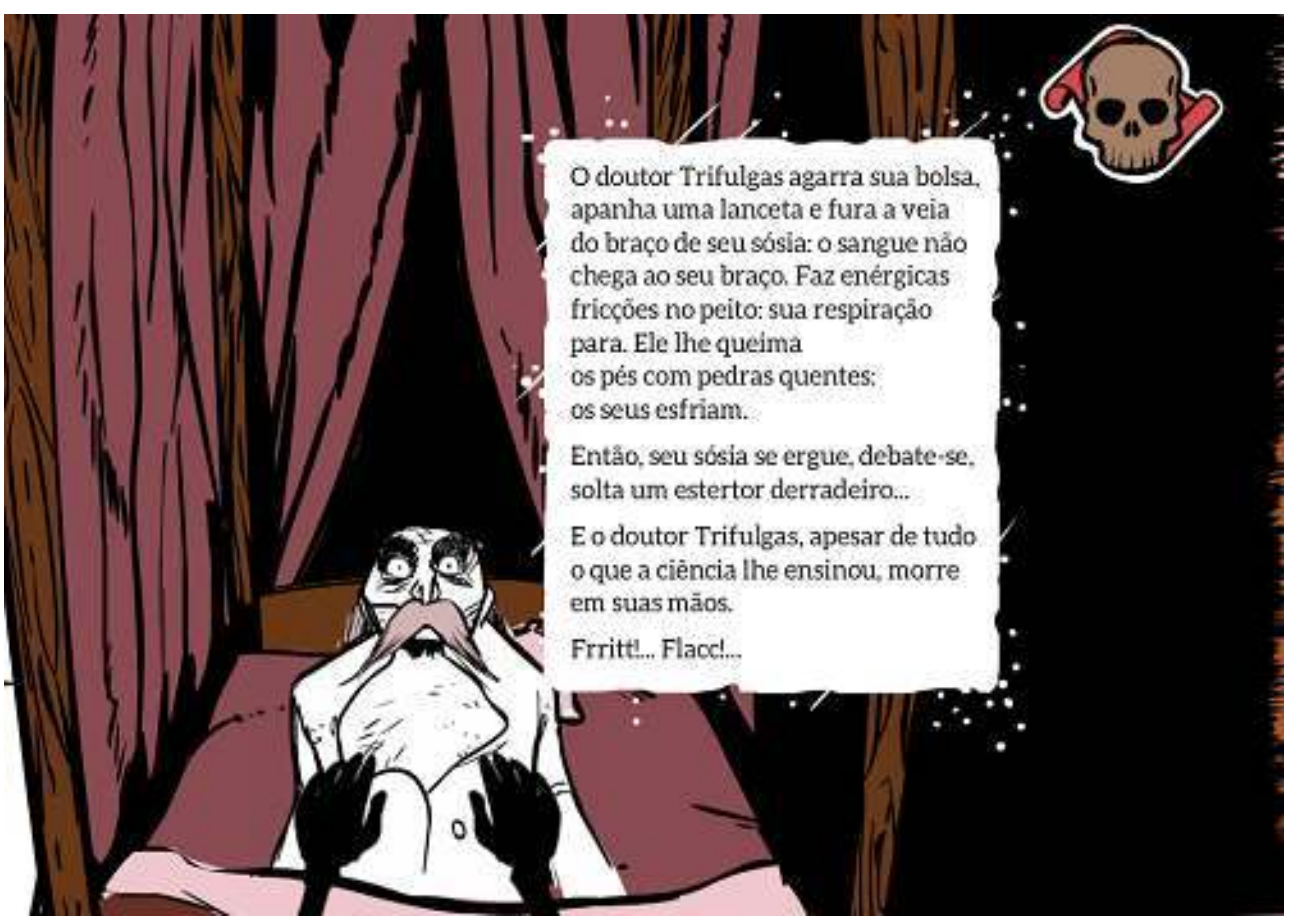




\section{Frritt-Flacc illustré}

15 responsable de la première traduction de Frritt-Flacc publiée au Brésil, dont la traductrice pour le portugais est Renata Calmon. C'est une belle édition illustrée par Alexandre Camanho $^{12}$. L'illustrateur est né en 1972, à São Paulo où il a étudié dessin et gravure, a obtenu un diplôme en Communication et s'est formé en Arts Plastiques à l'université. Depuis 2000, il réalise des illustrations pour des journaux, des revues et pour des maisons d'édition.

En rapport avec la culture française il a illustré en 2010 les Fables de La Fontaine, il a réalisé la couverture de La Tulipe noire d'Alexandre Dumas, des caricatures (Descartes...), des dessins du Marquis de Sade...

Dernièrement, Camanho s'est consacré aux livres pour enfants et il a remporté plusieurs prix d'illustration importants ${ }^{13}$, comme la seconde place du prix Jabuti 2015, obtenue par Os três ratos de Chantilly ${ }^{14}$, l'un des plus importants au Brésil. Il s'agit d'une adaptation libre, une version fabulée d'un fabliau picard du XIII ${ }^{\mathrm{e}}$ siècle assez connu, Les trois aveugles de Compiègne, attribué à Courtebarbe.

Frritt-Flacc est aussi un album où les protagonistes sont des animaux : Trifulgas est devenu un renard et les trois femmes, la fille, la femme et la mère du moribond, sont des oiseaux. Camanho a réalisé une lecture fabulée de l'original et il déclare qu'il a décidé de transformer les personnes en animaux, car ceux-ci apportent leurs caractéristiques aux humains et les enfants les perçoivent parfaitement d'une manière intuitive et non pas d'une manière objective.

Le choix d'un renard et des oiseaux pour les personnages de la nouvelle implique l'introduction d'une forte charge symbolique. Selon Pérez-Rioja (1984), dans l'imaginaire européen le renard représente la ruse et le comportement déloyal. Il symbolise aussi le Diable, surtout au Moyen Âge et c'est un personnage qui joue un rôle très important dans les fables et dans les traditions populaires. Le renard a eu toujours une double identité entre animal et humain. D'autre part, l'élection d'un oiseau pour représenter la fille, l'épouse et la mère du moribond constitue une réussite de l'illustrateur. Symbole de la spiritualité, face à l'attachement à la richesse de Trifulgas, l'oiseau est un porteur de présages. Bien que l'on ne puisse pas identifier clairement le type d'oiseaux, on dirait une poulette, une poule et une vieille poule, des animaux qui ne volent pas, ce qui montrerait leur enracinement au terrain, à la réalité quotidienne. De plus, leur antagoniste, le renard s'identifie au prédateur redouté des animaux de la basse-cour, présent dans les jeux traditionnels.

D'autre part, l'outil préféré de l'artiste pour créer ses dessins et illustrations est la plume à encre et comme procédé de gravure, le monotype. Il utilise aussi aquarelle, crayon Conté fusain, teinture extrait brou de noix, crayon, stylo-technique, vernis gomme-laque. Il explique sa technique.

Pour Frritt-Flacc, les illustrations ont été réalisées à traits de plume et rafales de vent chargés de brou de noix qui noircissent le papier.

Les personnages sont nés d'un tourbillon. Ils habitent pendant des jours et des nuits les rues sombres de Luktrop et je crois qu'une partie de moi est restée là ou qu'une partie de là reste toujours en moi ${ }^{15}$ (Verne, $2012: 69$ ). 
Dans la mise en page de l'œuvre, dans l'interaction entre texte et image, les éléments paratextuels, (couverture, pages de garde, etc.) sont importants et très significatifs. Dans la composition habituelle d'un livre, la deuxième et la troisième de couverture sont des pages vierges. L'originalité de cette publication réside dans le fait que la deuxième se déploie sur trois volets, avec typographie du titre et illustration, et dans la troisième de couverture, le titre en ocre couvre toute la page sur fond noir. De plus, l'illustration de la première de couverture (figure 9$)^{16}$ se poursuit à la quatrième (fig. 10). Si on les observe en parallèle on se rend compte du contraste qui prédomine dans l'ouvrage : le sombre sur la première et la clarté sur la quatrième.

Fig. 9

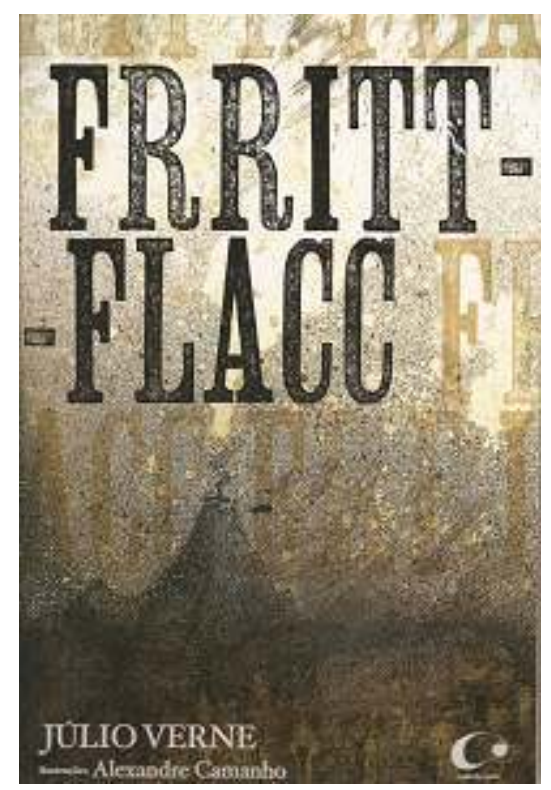

Fig. 10

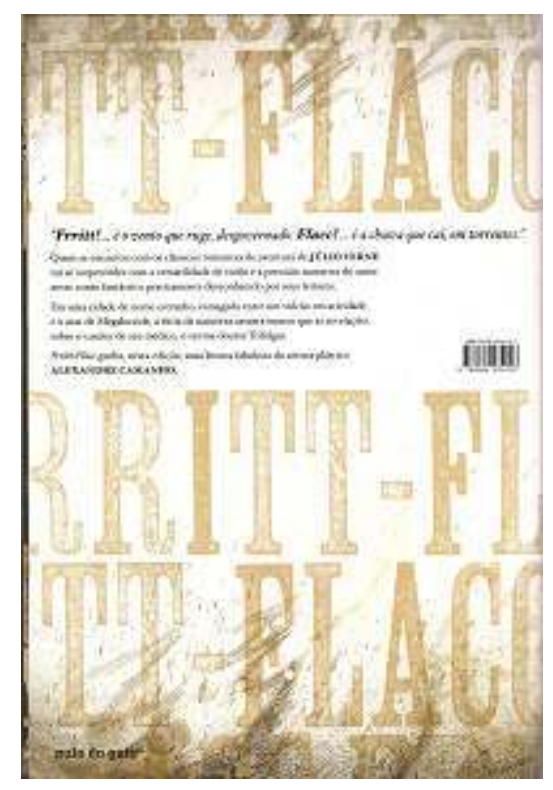


Toutes les illustrations du livre occupent la page entière, soit celle de droite (belle page), soit celle de gauche (fausse page). Leur orientation est verticale, sauf dans le cas des illustrations à double page représentant, d'abord, la ville de Luktrop (pp. 14-15), un paysage obscur illustratif, descriptif et non métaphorique. Une ligne horizontale sépare l'eau de la terre. C'est la côte volsinienne, les montagnes de Crimma et la mer de la Mégalocride. Luktrop est dessiné comme amalgame, réunion hétéroclite architecturale à silhouette imprécise presque effacée, avec le volcan au fond qui lance ses vapeurs sulfurées; ensuite la chambre du moribond (pp. 53-54) avec le lit, la chaise et le placard situés à gauche, et à droite sur la table, illuminé, le codex ouvert. L'image à double page qui suit est construite à partir de trois personnages (pp.40-41), le chien, Trifulgas et la vieille qui marchent à contresens (de droite à gauche). Cette image marque le renversement du temps et de l'espace ; le trajet initial (de Luktrop à Val Karniou) qui était parcouru dans le sens de gauche à droite, maintenant s'est renversé. On dirait que les personnages retournent en arrière, qu'ils reviennent au point de départ. Les deux dernières illustrations doubles sont, d'une part, la rafale mugissante (pp.12-13) qui « courbe les arbres de la côte volsinienne » dont Verne parle au premier chapitre; c'est l'image-résumé de cette tempête orageuse et bizarre aux tons ocre-jaunes. D'autre part, la tempête caractérisée par les vents violents et l'orage (figure 11) est reprise en double page à la fin du livre (pp. 66-67) ainsi que les masques fantomatiques qui l'accompagnent.

Fig. 11

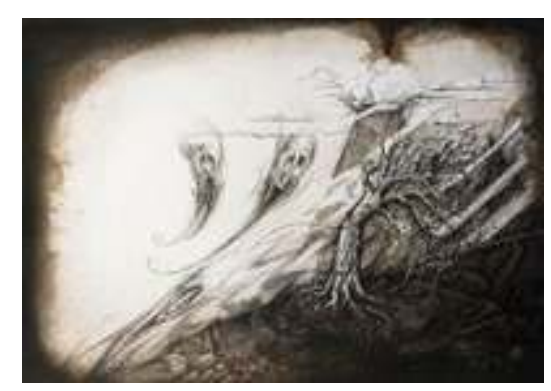

Le cadrage, c'est-à-dire la position que prend l'artiste par rapport aux objets, est très significative dans la composition de l'image. Presque toutes sont dessinées sous un angle de vue frontal Cela signifie que le spectateur se trouve au même niveau que la représentation artistique. Il existe uniquement deux illustrations (figures 12 et 13), où l'angle de vue est en plongée et se correspondent avec la représentation de l'étrange maison du Six-Quatre. 
Fig. 12

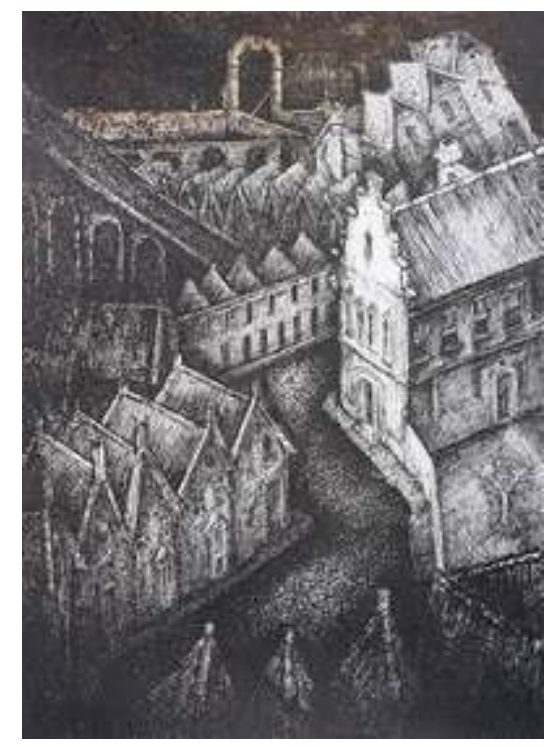

Fig. 13

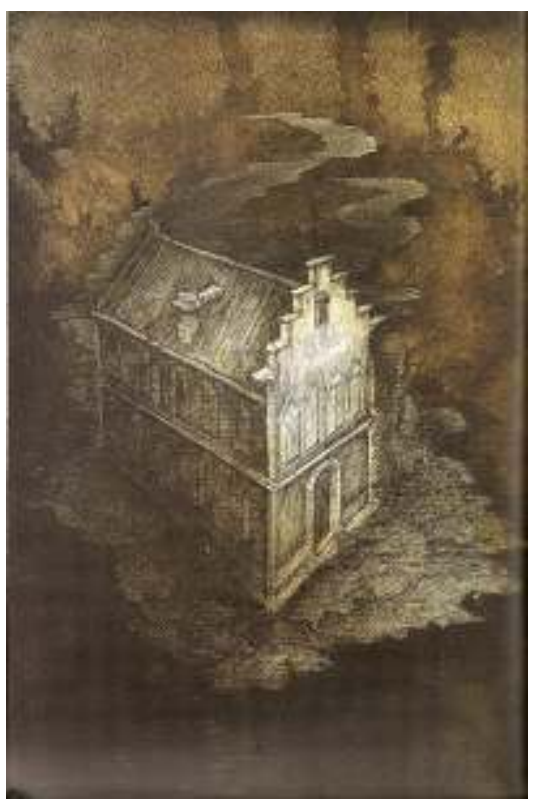

Ici l'artiste/le lecteur-observateur se situe plus haut que l'objet. Il oriente donc le regard vers le bas (en plongée). Ce cadrage donne une certaine impression de détresse, de danger ou de solitude qui convient très bien à l'histoire vernienne ${ }^{17}$.

Nous avons déjà ébauché avant que le contraste noir-blanc préside cet ouvrage. Les six chapitres principaux de la nouvelle sont imprimés sur feuille à fond noir avec le texte à caractères ocre-jaune. Par contre le septième, le dernier chapitre qui fonctionne comme épilogue, voire comme conclusion inexpliquée, contraste avec toute l'histoire car les pages ici sont toutes blanches et la typographie noire. Il faut se rappeler que dans ce chapitre, Verne ne fournit pas d'explication logique et maintient le lecteur dans sa confusion. Selon Ana Alonso (2007 : 148), l'auteur a décidé de construire son récit selon les règles du genre fantastique le plus pur; un genre qui demeure en territoire 
d'incertitude et d'hésitations où la réponse aux questions posées par le lecteur n'existe plus.

Le seul personnage illustré de ce chapitre blanc est le fidèle chien Hurzof, immortel et immortalisé sur un portrait ovale ${ }^{18}$. Cette image ovale qui regarde vers la gauche et ferme le récit (figure 14) ainsi que l'autre image ovale ouvrant le récit, qui regarde vers la droite (figure 15) et se trouve sur la page de titre, sont l'avers et le revers de la même médaille.

Fig. 14

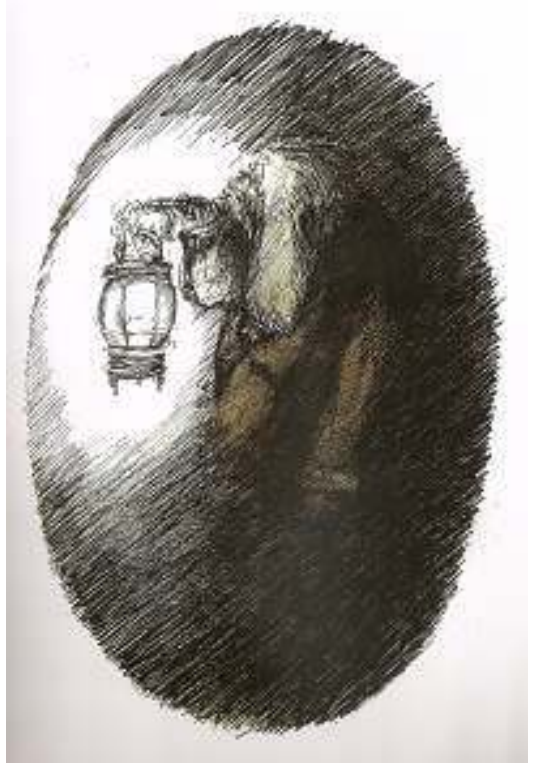

Fig. 15

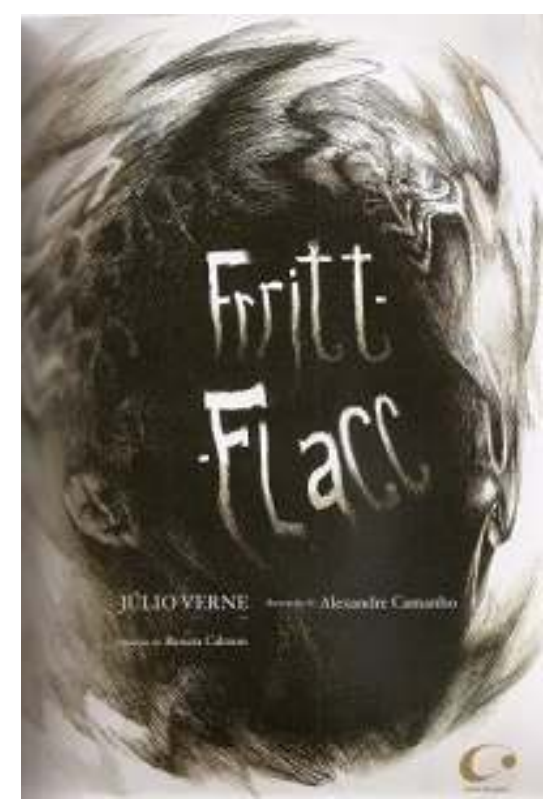

21 Cette illustration de la page de titre suggère un visage qui regarde vers la droite et indique le trajet de lecture à suivre. À l'intérieur se trouve le titre de l'ouvrage avec typographie à traits fantastiques, un peu fantomatiques. On y apprécie clairement trois masques, symboles de la médiation entre le monde réel et le surnaturel. Ces trois visages 
sont représentés deux de face, sur la diagonale, et un troisième de profil, dans la partie droite de l'illustration. Les trois ont la bouche ouverte, comme s'ils riaient dans l'au-delà. Celui de gauche ressemble à un ancien masque brésilien. Cette représentation grotesque du visage, le nez en forme de flèche avec la pointe vers le bas, est une caractéristique très particulière de l'illustrateur.

D'autre part, on observe sous un fond noir, tout au moins cinq crânes, plutôt des cavités orbitaires vides, qui font le lien avec les deux masques qui regardent le lecteur comme signe révélateur de ce qui l'attend.

Dans le sixième chapitre Verne introduit deux thèmes très importants : la présence de l'insolite et la rencontre avec le double, et cela a aussi son reflet dans les deux illustrations qui suivent.

La première (figure 16) est cadrée en contre-plongée ; ici l'observateur est un peu plus bas que la scène et doit orienter son regard vers le haut. Ce cadrage permet de mettre en valeur Trifulgas qui monte par l'escalier et qui s'approche de cette porte inquiétante derrière laquelle on ne sait pas ce qu'il va trouver.

Fig. 16

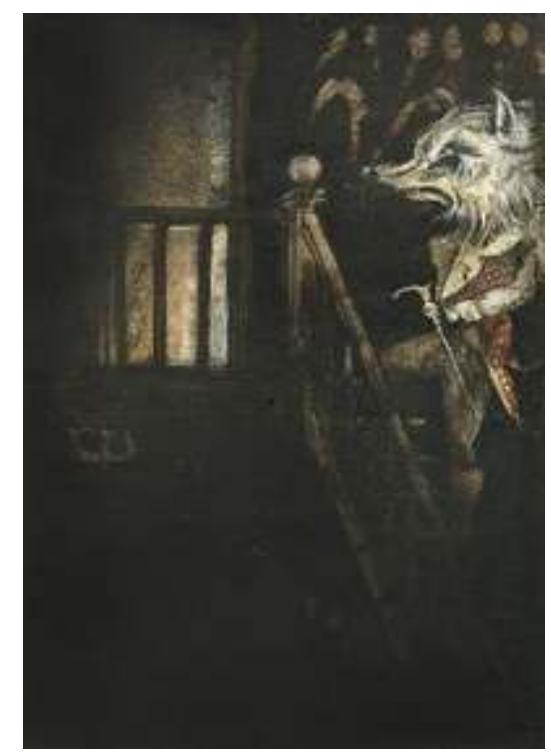

Les deux éléments illuminés sur la scène sont Trifulgas, la porte de la chambre et la boule de la rampe, objets parfaitement détaillés par Verne dans la nouvelle.

Sur l'image, l'arrière-plan a moins d'importance, même si les «âmes du monde souterrain " sont là, à l'affut. On y aperçoit six silhouettes qui flottent en haut ainsi qu'une cavité orbitaire au-dessous du palier.

L'illustration suivante (figure 17) narre la rencontre avec le double. L'artiste a su montrer l'étonnement dans le regard de Trifulgas car il vient de se rencontrer avec son double, qui est sur le point de mourir. 
Fig. 17

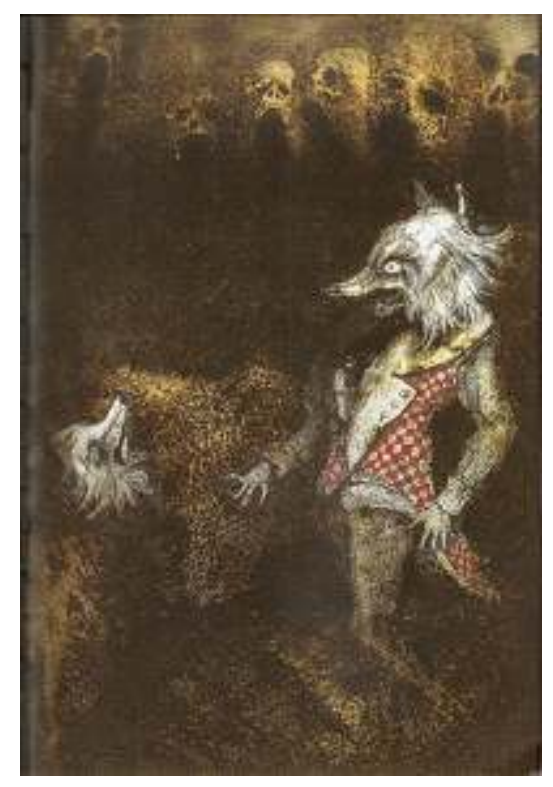

Ce moment de la mort imminente est très bien réussi. Pour la narration visuelle de l'instant précis, Camanho s'est servi des draps qui, sous l'apparence d'un fantôme, vont porter dans leurs bras le moribond. La scène est accompagnée de fantasmes, de spectres, de masques surnaturels, la bouche ouverte, qui flottent dans la partie supérieure de l'illustration. Encore six crânes, un peu flous, couvrent toute la partie supérieure pour fermer la scène, et indiquent que la sortie est impossible.

Tout est en train de disparaitre et la disparition commence par ce qui est collé à la terre, parce que tout ce qui est terrestre, humain, passager, fini, en définitive... mortel.

Nous avons déjà montré quelques exemples de la narration visuelle qui sont aussi des éléments présents dans la nouvelle, comme le double ou les aspects relatifs au conte moral. Pour finir, on voudrait souligner d'autres traits significatifs qui sont en rapport avec le cryptage, le mystère et la sonorité de l'onomatopée du titre.

Pour représenter le flacc de la pluie ainsi que le frritt du vent, Alexandre Camanho a créé deux métaphores visuelles magnifiques, deux images en même temps tactiles et sonores. Sur l'illustration de la pluie (figure 18), la partie supérieure, foncée, évoque des nuages ocre-jaunes, moutonnés et menaçants, qui versent de l'eau en abondance. Il s'agit d'un orage rocheux, dense et matériel. 
Fig. 18

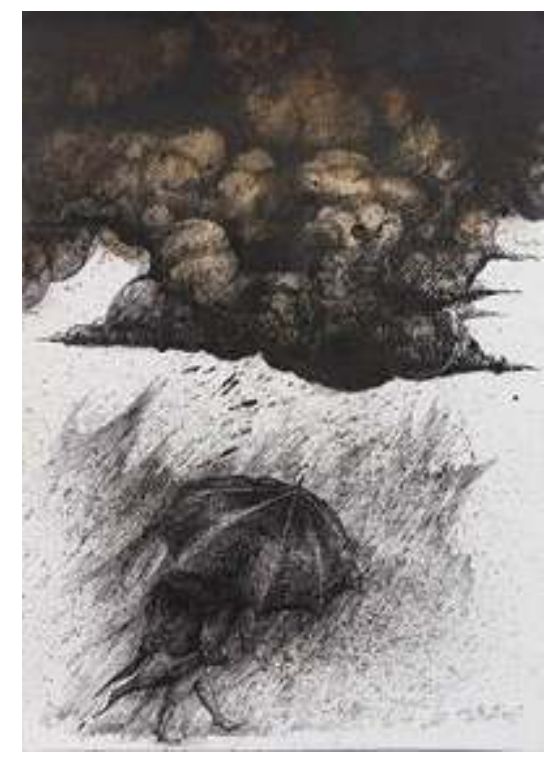

De la même manière que Verne utilisait littérairement les occlusives, en particulier le son occlusif vélaire sourd $[\mathrm{k}]$ pour transmettre la sonorité de la pluie, l'artiste évoque parfaitement la sonorité du flacc, clac, à travers l'image de ces nuages rocheuses.

La clarté est sur la partie inférieure où l'on devine la présence de quelqu'un qui se protège sous un parapluie et marche sous la tempête noire et blanche.

Ce personnage présente une forte similitude avec l'étrange violoniste de l'image qui précède dans l'histoire (figure 18), parce que sous le parapluie, on identifie les pointes de sa jaquette (qui ressemblent fortement à la langue bifide du serpent). Les deux illustrations sont donc complémentaires, la pluie-flacc, située sur la belle page, celle de droite, et à l'opposé, le vent-frritt, placé sur la fausse page, page pair.

Cette illustration venteuse (fig. 19) est celle qui ouvre la nouvelle, et est aussi l'image choisie pour fermer l'ouvrage parce qu'elle est répétée.

\section{Fig. 19}

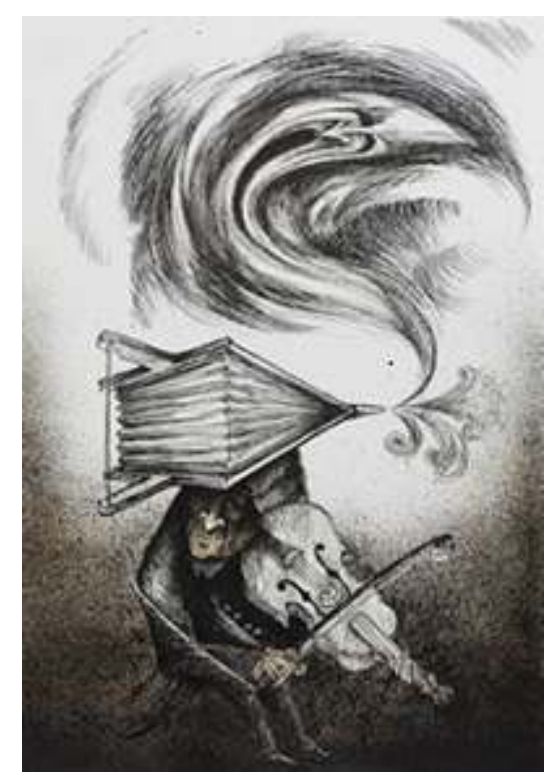



prédominent sont le blanc, le gris, le gris foncé, et l'ocre-jaune. C'est une image tactile et sonore du vent représenté par deux éléments : le violon et le soufflet.

$\mathrm{Au}$ milieu, le soufflet chasse une grande quantité d'air d'une manière brusque et violente. Cette poussée sans contrôle est reflétée au moyen de l'air serpentant qui monte sinueusement. Ce S, serpent symbolique présent dans toutes les cultures, enserre le masque fantomatique, auquel on s'est déjà référé, qui a la bouche ouverte et qui s'allonge en sortant de l'appareil.

C'est aussi le $\mathrm{S}$ du serpent de l'iconographie chrétienne qui symbolise le mal, le péché ainsi que l'avènement de la mort. C'est l'image du Diable, du pouvoir du Malin ; le serpent évoque l'astucieux tentateur qui induit au pêché. Dans le monde gréco-romain, cet ophidien était associé au culte d'Asclépios-Esculape, dieu de la médecine et emblème universel de l'ancienne pharmacopée et de la pharmacie actuelle (Pérez-Rioja, J.A. 1984, p. 385). À travers ce mouvement ondulant, Camanho a intégré parfaitement dans l'histoire visuelle cet élément essentiel, le codex, ancienne pharmacopée, dont la fonction dans la nouvelle vernienne est d'annoncer au lecteur que la mort approche.

En même temps, le soufflet expulse par la tuyère trois courtes rafales qui se déploient l'une vers l'horizontale et les deux autres vers le bas. Ce soufflet, qui partage l'image en deux, est placé sur la tête du violoniste, celui-ci situé dans la partie inférieure. Le visage (bouche fermée) et la main droite de l'instrumentiste se détachent de l'obscurité par l'utilisation de la couleur ocre-jaune. De sa main droite, en même temps qu'il fait glisser l'archet sur le violon, le violoniste actionne le soufflet, car les deux éléments tactiles et sonores (soufflet et violon) sont liés par une corde.

Mais non seulement le violon et le soufflet sont attachés, mais aussi la partie supérieure et la partie inférieure de l'illustration. La figure ondulante et fantomatique est, en définitive, le visage du violoniste. Les deux visages se reconnaissent par le nez identique. Le $\mathrm{S}$, le soufflet et le violon sont illuminés de la même manière.

Dans cette illustration, le blanc occupe la partie supérieure; c'est la zone du vent qui monte sinueusement. Par contre, les tonalités sombres, ocre et jaunes se rassemblent dans la partie inférieure.

Nous sommes devant l'image la plus réussie de l'artiste et la plus évocatrice qui, en plus, englobe en elle-même l'étrange maison du Six-Quatre (le six, forme de l'air serpentant, et le quatre, dessiné par la position du violoniste).

En définitive, la lecture de l'image fournit au lecteur beaucoup plus d'information que le texte qui l'accompagne: «Frritt!... c'est le vent qui se déchaîne.»(p.7). Avec cette illustration initiale, l'artiste suggère, mais aussi anticipe, la thématique et quelques éléments fondamentaux de la nouvelle : le fantastique, le double, le S (le Malin, le mal, le médecin, la pharmacopée ou le codex, le Six-Quatre). De plus, la même image répétée a été choisie pour clore ce Frritt-Flacc illustré brésilien, comme s'il s'agissait du résumé visuel de la nouvelle de Jules Verne.

\section{Considérations finales}

Les éléments qui conforment les deux Frritt-Flacc en images et que nous avons présentés, gardent des liens avec la mémoire culturelle occidentale et s'insèrent parfaitement dans l'imaginaire collectif de nos sociétés. 
De la même manière que Trifulgas, après avoir parcouru un trajet, semble revenir au point de départ, nous reprenons l'article de Carmelina Imbroscio (2013). Le ton ironique ainsi que le langage vernien repéré n'a pas de place dans le livre animé ni dans le livre fabulé. C'est parce que le lecteur visé est, on l'a déjà dit, un public jeune, formé par des enfants, à partir de six ans, et des adolescents. Les sons, les bruits, les onomatopées, les allitérations de la nouvelle se manifestent d'une manière différente, parce que les formats le sont aussi. La musique fait partie de la création de l'atmosphère sinistre dans l'application ainsi que les sons et les bruits produits par les éléments naturels et artificiels, par le chien et les autres personnages, qui servent aussi à mettre l'accent sur les instants les plus importants de l'intrigue.

Ainsi, les thèmes principaux de la nouvelle vernienne y sont-ils présents, bien que de manière différente parce que StoryMax et Pulo do Gato doivent s'adapter à leurs publicscible et aux deux formats visuels choisis : la nature qui se déchaine avec le vent, la pluie, la tempête orage et le volcan; le fantastique et le surnaturel à travers les âmes qui flottent, le Malin et le double; les espaces extérieurs imaginaires et le trajet menaçant, à Luktrop, dans la maison du Six-Quatre ou le clocher de Sainte-Philfilène; les espaces intérieurs conformés par la lumière, le décor, ainsi que par les objets, comme le lit, le codex ; nous ne devons pas oublier les personnages : les trois femmes, Trifulgas et le chien Hurzof; de même les autres éléments sonores et visuels : bruits, allitérations, symétrie, répétitions, distance, perspective, etc.

Dorénavant, il faudra prendre en considération ces deux curieuses adaptations qui conviennent très bien au récit le plus atypique et original de Jules Verne.

\section{BIBLIOGRAPHIE}

ALonso, Ana (2007). « Julio Verne en territorio fantástico. Análisis de Frritt-Flacc », Epos, no 23, pp. 139-149.

APARICI, Roberto \& GARCíA MATILLA, Agustín (1989). Lectura de imágenes. $2^{\mathrm{e}}$ éd. Madrid : Ediciones de la Torre.

IMBRoscio, Carmelina (2013). « Du tarissement de l'âme à la dissolution du corps. Le thème de la mort dans Frritt-Flacc ». À la vie, à la mort !, Revue Jules Verne, no36, pp. 88-95.

PÉREZ-RIOJA, José Antonio (1984). Diccionario de símbolos y mitos. 2e éd. Madrid : Tecnos.

RADULESCU, Mihaela (2014). « La narrativa visual » [entrée de blog] [on-line] [disponible le 30/09/2018] <URL : http://comunicaciongrafica-pucp.blogspot.com/2014/05/la-narrativavisual.html>

VERNE, Jules (1886). Frritt-Flacc, avec Un Billet de loterie. Paris : J. Hetzel, col. Bibliothèque d'Éducation et de Récréation, pp. 189-198. Illustrations George Roux.

VERNE, Júlio (2012). Frritt-Flacc. São Paulo : Pulo do Gato. Traduction en portugais de Renata Calmon. Illustrations : Alexandre Camanho. 
VERNE, Júlio (2016). Frritt-Flacc, livre-app trilingue portugais, espagnol et anglais. StoryMax, Novozymes et SESI PR-Paraná, Brésil (éd.), <URL : http://www.storymax.me/frritt-flacc/> VIALA, Laurie (2005). « Les Voyages extraordinaires : une invitation aux images », in Jean-Pierre Picot, Christian Robin (dir.). Jules Verne : Cent ans après. Actes du colloque de Cerisy. Dinan : Terre de Brume, pp. 109-122.

\section{NOTES}

1. «Este livro foi publicado pela Editora Pulo do Gato em 2012, após ter dado a volta ao mundo em 80 dias num balão até encontrar o conto Frritt-Flacc. »

2. Sur ce sujet et à propos de Jules Verne, l'article de Laurie Viala (2005) est très illustratif.

3. Frritt-Flacc, livre-app trilingue pour IOs et Android, réalisée en collaboration entre Novozymes, StoryMax et SESI PR-Paraná, Brésil (Ed), supportée par CRBio-Paraná, Brésil, 2016. <URL http:// www.storymax.me/frritt-flacc/>. Toutes les adresses électroniques citées dans cet article étaient disponibles le 12/10/2018. >. Toutes les adresses électroniques citées dans cet article étaient disponibles le 12/10/2018.

4. Il a remporté le Selo Distinção Cátedra 10-2016 décerné par l'Instituto Interdisciplinar de Leitura PUC-Rio et la Cátedra Unesco de Lecture PUC-Rio. De même, il a été reconnu par des spécialistes en lecture numérique du Royaume Uni avec l'Opening Up Reading Digital Fiction Award.

5. «Frritt-Flacc app book is a classic literary work from the French writer Jules Verne. Its plot, in the form of suspense, revolves around social differences, addressing human indifference to the problem and the punishment of poverty today. " <URL: https://www.linkedin.com/pulse/ unesco-seal-distinction-chair-10-2016-pedro-luiz-fernandes>

6. L'organigramme de StoryMax, le nom et la carrière des illustrateurs et développeurs peuvent être consultés sur https://storymax.me/en/. Selon les crédits de l'application Frritt-Flacc, la traduction du texte en portugais est attribuée à Daniel Cariello, le texte anglais à Gitanjali Patel ainsi que la traduction des paratextes, ceux-ci en collaboration avec Alyne Azuma. La version en espagnol est due à Sergio Molina et Rubia [Prates] Goldoni, et la traduction des paratextes en castillan est de José Muniz Jr.

7. Bande annonce disponible sur https://www.youtube.com/watch ? $\mathrm{v}=$ toyEwusIAfI\&feature =youtu.be, livre-app seulement en portugais.

8. Bande annonce du livre-app Frritt-Flacc disponible sur https://www.storymax.me/frrittflacc.

9. Contenus additionnels très brefs, sur l'auteur, considéré le père « da literatura moderna de ficção científica ", " of modern scientific literature ", " de la literatura moderna de ciencia ficción »; et sur son œuvre, où une erreur se répète dans les trois langues: «Frritt-Flacc, reeditado em conjunto com outras duas histórias curtas en 1886, dois anos após ter sido originalmente apresentado na revista Le Figaro Illustré ». Il est bien connu que la nouvelle fut publiée en 1886, chez Hetzel dans un volume simple avec le roman Un Billet de loterie, et dans un volume double auquel on ajouta un autre roman, Robur le conquérant.

10. Roberto Aparici et Agustín García Matilla (1989: 90-91) recueillent dans une grille le symbolisme des couleurs d'après différents auteurs.

11. Nous remercions vivement Mme Samira Almeida co-fondatrice et éditrice de StoryMax, qui nous a autorisées à reproduire les images du livre-application, faites à partir de capture d'écran.

12. Les créations artistiques d'Alexandre Camanho sont disponibles sur son blog http:// alecamanho.blogspot.com/ Le post relatif aux illustrations de Frritt-Flacc (30/01/2014) sont sur http://alecamanho.blogspot.com/2013/07/frritt-flacc.html. De même, la page de titre ainsi que 
les pages 6 à 13 de Frritt-Flacc se trouvent dans la plate-forme d'édition électronique Issu. https:// issuu.com/pulodogato/docs/frritt_flacc_issuu.

13. Voir l'entrevue d'Alexandre Camanho avec Maria José Petri dans le programme de télévision ArteLetra émis en 2015, dans la TV São Judas de Brasil, disponible sur le lien de l'artiste http:// alecamanho.blogspot.com/2017/02/tv-sao-judas-entrevista-serie-infantil.html. Une autre, de 2016 sur les prix remportés est disponible sur https://www.youtube.com/watch ?v=VD5VBwYfew.

14. Camanho dessinant les trois souris, travail réalisé pour être publié chez Pulo do Gato. Disponible sur https://www.youtube.com/watch ?v =E-fBUAjxtw4.

15. «Para Frritt-Flacc, as ilustrações foram feitas a penadas e rajadas de ventos carregadas de extrato de nogueira que enegreceram os papéis; assim os personagens nasceram de um redemoinho. Habitei por dilatados dias as tuas sombrias de Luktrop, e creio que algo de mim tenha ficado lá ou que algo de lá em mim permaneça » (Verne, 2012 : 69).

16. Nous tenons à remercier vivement $\mathrm{M}$. Leonardo Chianca, éditeur et membre de la maison d'édition Pulo do Gato, qui nous a autorisées à reproduire les illustrations pour la réalisation de ce travail.

17. Il faudra rappeler que dans le livre-application, la maison du Six-Quatre était détachée par l'utilisation du rouge vif.

18. The Oval Portrait (1842) est le titre d'une micro-nouvelle fantastique d'Edgar Allan Poe, écrivain qui a eu de l'influence sur Jules Verne dans la création de Frritt-Flacc.

\section{RÉSUMÉS}

Nous nous proposons dans cet article d'analyser les deux dernières parutions au Brésil de la nouvelle de Jules Verne Frritt-Flacc (1884). La première traduction brésilienne de ce récit a été publiée, en 2012, par la maison d'édition Pulo do Gato, illustrée par Alexandre Camanho tandis que la deuxième, datée de 2016, est une application mobile animée conçue par Novozymes, StoryMax et SESI-PR, téléchargeable gratuitement. Nous ferons aussi une analyse plus détaillée de la forme et des contenus de ces deux manières de viser un public-cible, majoritairement jeune, avec ces deux formats si spécifiques. On y montrera comment grâce aux images et aux illustrations on fait passer l'intrigue et les aspects formels de la nouvelle vernienne. Notre objectif est celui d'explorer un autre langage qui complète l'œuvre originale française et de contribuer ainsi à mieux la faire connaître et à élargir son public en mettant dans les mains des lecteurs deux formules différentes de se rapprocher de ce récit fantastique.

In this paper we intend to analyze the last two versions of Jules Verne's Frritt-Flacc published in Brazil. The first Brazilian translation of Verne's story was published in 2012 by Pulo do Gato and it was illustrated by Alexandre Camanho, while the second version was released in 2016 as a free app-book and it was created by StoryMax with Novozymes and SESI-PR.

Our research explores how the above-mentioned Frritt-Flacc versions attempt to catch the imagination of their target young audiences through a series of specific themes and formal feature. The main objective of our project is precisely to investigate how narrative devices and visual language expand and redefine the scope and meanings of the original French work under discussion, thus, helping readers and critics to have a deeper, more significant understanding of Verne's rich fantasy story. 
INDEX

Mots-clés : Verne (Jules), Frritt-Flacc, adaptation, illustration, livre app

Keywords : Verne (Jules), Frritt-Flacc, adaptation, illustration, app-books

\section{AUTEURS}

MARÍA-LOURDES CADENA

Université de Saragosse

mlcadena[at]unizar.es

MARÍA-TERESA BAREA

Université de Saragosse

tbarea[at]unizar.es

T3AXEL 